\title{
Philonsorbonne
}

$5 \mid 2011$

Année 2010-2011

\section{Pluralisme logique, tolérance et empirisme}

\section{Pierre WAGNER}

\section{(2) OpenEdition}

Journals

Édition électronique

URL : https://journals.openedition.org/philonsorbonne/353

DOI : 10.4000/philonsorbonne.353

ISSN : 2270-7336

\section{Éditeur}

Publications de la Sorbonne

\section{Édition imprimée}

Date de publication : 15 mai 2011

Pagination : 97-117

ISBN : 978-2-85944-680-2

ISSN : 1255-183X

\section{Référence électronique}

Pierre WAGNER, «Pluralisme logique, tolérance et empirisme », Philonsorbonne [En ligne], 5 | 2011, mis en ligne le 03 février 2013, consulté le 08 juin 2021. URL : http://journals.openedition.org/ philonsorbonne/353 ; DOI : https://doi.org/10.4000/philonsorbonne.353

(c) Tous droits réservés 


\title{
Pluralisme logique, tolérance et empirisme
}

\author{
Pierre Wagner \\ Maître de conférences \\ à l'université Paris 1 Panthéon-Sorbonne
}

\section{Qu'est-ce que le pluralisme logique?}

La logique est généralement conçue comme l'étude des principes du raisonnement, ramenée en fait à l'étude des règles qui caractérisent les inférences et les énoncés formellement valides. Bien que la question du statut épistémologique de ces règles, comme celle de leur justification et de leur usage légitime soit, depuis longtemps, très disputée, une longue tradition tient les règles et les lois de la logique pour universelles et immuables. Le pluralisme logique remet en question cette universalité en énonçant qu'il n'y a pas une logique, mais plusieurs. Que peut-on vouloir dire par là ? Imaginons qu'à l'occasion d'un raisonnement, je fasse usage de la loi de la double négation en inférant par exemple «il pleut» de la prémisse «il est faux qu'il ne pleut pas » et que mon interlocuteur refuse de reconnaître l'inférence comme logiquement valide, protestant que la prétendue loi de la double négation n'est pas une loi logique universellement valide. Je peux tenter d'engager avec lui une discussion sur cette question, afin d'essayer de le convaincre en avançant raisons et arguments, mais si mon argumentation s'appuie sur des raisonnements dans lesquels je fais à nouveau usage de lois logiques dont il ne reconnaît pas la validité universelle, il est bien possible que nous ne réussissions pas, en définitive, à nous accorder. Dans une telle situation, je puis être tenté de rejeter les allégations adverses dans la catégorie de l'irrationnel, de l'illogique ou de la mauvaise foi, mais j'adopte alors une stratégie par laquelle je renonce tout simplement à trouver avec mon interlocuteur un quelconque accord fondé sur des règles ou des lois logiques. Le pluralisme logique consiste à prendre acte d'une divergence possible sur les principes mêmes du raisonnement et à reconnaître du même 
coup qu'il n'y a pas une mais plusieurs logiques. Dans le cas présent, il ne s'agit pas d'une divergence relative à l'application ou à l'interprétation de règles dont la validité serait admise par ailleurs : la divergence porte sur la détermination des règles elles-mêmes.

Une justification possible du pluralisme logique consiste à dire que le concept d'inférence valide - que les règles logiques servent à déterminer n'a pas, dans la langue usuelle, un sens si précis qu'il n'ouvre la possibilité de caractérisations divergentes lorsqu'on tente de lui donner un sens parfaitement exact. Selon une autre interprétation ou justification possible, s'il n'existe aucune logique universelle, c'est parce que les règles de la logique sont contextuelles : en dépit du fait que ces règles, en tant qu'elles relèvent de la logique, sont formelles par définition (au sens où elles ne dépendent pas de l'objet auquel elles sont appliquées), un seul et même ensemble de règles ne s'applique pas de manière uniforme à tout raisonnement. En adoptant cette position, on conserve cependant l'idée d'une normativité indépendante de tout choix possible: dans tel contexte, c'est telle logique définie par tel ensemble de règles qui s'applique, et nulle autre; dans tel autre contexte, tel autre ensemble de règles.

Le point de vue de Carnap est différent et plus radical dans son rejet d'une normativité logique prétendument indépendante de toute stipulation : il consiste à soutenir qu'aucun critère ne permet de déterminer, parmi les multiples logiques que l'on pourrait imaginer formuler sous la forme d'un ensemble de règles, celle qui est correcte et celles qu'il convient de rejeter comme incorrectes. En outre, ce ne sont pas des différences de contexte qui sont à la base de son rejet de l'universalisme logique. Selon le pluralisme carnapien, il n'existe tout simplement rien par rapport à quoi pourrait être définie une notion de correction ou de vérité en logique, si ce n'est un ensemble de règles stipulées, qui n'ont en elles-mêmes aucun caractère de nécessité. Carnap ne soutient donc pas seulement qu'il existe plusieurs logiques, mais également que choisir l'une d'entre elles relève d'une pure décision, c'est-à-dire d'une stipulation - bien que le choix que l'on puisse faire à cet égard ne soit pas nécessairement arbitraire pour autant.

La question du pluralisme logique fait l'objet, dans la philosophie de la logique actuelle, de nombreux débats ${ }^{1}$. Nous nous intéresserons ici surtout à l'interprétation qu'a donnée Carnap de cette idée à partir des années 1930 , interprétation à laquelle il est fait référence dans la plupart des discussions contemporaines. Elle est à la base d'une forme d'empirisme propre à cet auteur.

1. Cf. par exemple J. C. Beall et G. Restall, Logical Pluralism, Oxford, Oxford University Press, 2006 ; G. Russell, « One True Logic ?», Journal of Philosophical Logic, 37, 2008, p. 593-611; P. Köllner, "Truth in Mathematics : The Question of Pluralism », in O. Bueno et O. Linnebo (éds.), New Waves in Philosophy of Mathematics, Basingstoke, Palgrave Macmillan, 2009, p. 80-116; J.-Y. Beziau (éd.), Is Logic Universal ?, numéro spécial de la revue Logica Universalis, 4, 2010 ; M. Eklund, «The Multitude View on Logic », in G. Restall et G. Russell (éds.), New Waves in Philosophical Logic, Basingstoke, Palgrave Macmillan, à paraître. 
Historiquement, une situation de divergence relative à la question des principes du raisonnement s'est effectivement produite à plusieurs reprises, par exemple après que les paradoxes apparus dans la science mathématique, réputée la plus sûre, ont requis un examen précis de ses principes, et que Brouwer a proposé une analyse du raisonnement mathématique qui récusait l'usage généralisé de la loi du tiers exclu et de la loi de la double négation ${ }^{2}$; ou encore, lorsque l'analyse que Russell et Whitehead avaient proposée de l'implication matérielle dans les Principia Mathematica ${ }^{3}$ fut critiquée par C. I. Lewis, qui introduisit le concept d'implication stricte et une série de systèmes axiomatiques dont chacun caractérisait un concept d'implication différent ${ }^{4}$. L'opposition entre des conceptions divergentes de la logique, qui pouvaient entraîner la reconnaissance de lois et de règles logiques différentes, fut particulièrement virulente au cours des années 1920 qui virent se confronter, sur la question du fondement des mathématiques, d'un côté les partisans de l'intuitionnisme de Brouwer et de l'autre l'école formaliste de Göttingen, dont Hilbert était alors la figure dominante ${ }^{5}$. Les formalistes entendaient préserver les mathématiques de la mutilation qu'impliquaient, à leurs yeux, les restrictions dont l'intuitionnisme préconisait l'application à certaines lois logiques comme le tiers exclu. Vers la fin des années 1920, Carnap s'engagea lui aussi dans une recherche sur les fondements des mathématiques, en procédant dans un premier temps selon une démarche tout à fait caractéristique de son mode de pensée irénique : loin de prendre parti pour le logicisme de Frege ou de Russell, pour le formalisme de Hilbert, pour l'intuitionnisme de Brouwer ou pour la philosophie des mathématiques et de la logique du Tractatus logicophilosophicus de Wittgenstein, il chercha une solution capable de répondre aux difficultés qu'avait soulevées chacun de ces auteurs et susceptible de concilier les différents points de vue en présence. À partir de 1927, il s'engagea ainsi dans un vaste programme de recherche sur la logique et les fondements des mathématiques dont témoigne notamment un long manuscrit retrouvé dans ses archives et publié en $2000^{6}$. Ce manuscrit, resté inachevé, montre également à quelles apories ces recherches logiques aboutirent après

2. Cf. L. E. J. Brouwer, «Qu'on ne peut pas se fier aux principes logiques » (1908), trad. J. Largeault, in J. Largeault (éd.), Intuitionisme et théorie de la démonstration, Paris, Vrin, 1992, p. 18-23.

3. B. Russell et A. N. Whitehead, Principia Mathematica, 3 vol. Cambridge, Cambridge University Press, 1910-1913; 2 éd. 1925-1927.

4. Cf. C. I. Lewis, A Survey of Symbolic Logic, Berkeley, University of California Press, 1918, chap. V. C. I. Lewis et C. H. Langford, Symbolic Logic, New York, Century Company, 1932. Cf. aussi C. I. Lewis, «Alternative Systems of Logic », The Monist, 42, 1932, p. 481507, reproduit dans J. D. Goheen et J. L. Mothershead Jr. (éds.), Collected Papers of Clarence Irving Lewis, Stanford, Stanford University Press, 1970, p. 400-419.

5. Sur cette opposition, cf. les textes de Brouwer, Hilbert, Weyl et Bernays traduits par Jean Largeault in J. Largeault (éd.), Intuitionisme et théorie de la démonstration, Paris, Vrin 1992.

6. R. Carnap, Untersuchungen zur allgemeinen Axiomatik [Recherches pour une axiomatique générale], éd. Th. Bonk et J. Mosterin, Darmstadt, Wissenschaftliche Buchgesellschaft, 2000. 
que Carnap y eut travaillé pendant plusieurs années, jusqu'au tout début des années 1930. Ce qui nous intéresse ici n'est pas tant le détail des raisons pour lesquelles le projet échoua ${ }^{7}$ que le tournant radical que Carnap choisit d'opérer en conséquence, à partir de 1932, dans ses recherches relatives au problème du fondement des mathématiques, tournant qui consista dans l'adoption d'une forme de pluralisme logique et du principe de tolérance. Jugeant stériles les débats qui portaient sur la justification des principes de la logique, qui, en définitive, renvoyaient invariablement à des positions philosophiques divergentes et inconciliables, Carnap défendit alors une position qui consistait à renoncer tout à fait à toute tentative de fondation de ces principes dans une objectivité ou une factualité qui eût permis de faire le départ entre principes corrects et incorrects. Il proposa de reconnaître que des principes logiques sont adoptés non en raison de leur caractère véridique mais pour des raisons pragmatiques de commodité, de convenance ou d'opportunité. De ce point de vue, il n'était plus question pour lui de chercher à concilier des conceptions divergentes des fondements de la logique et des mathématiques. La question de la correction des principes logiques ne se posait tout simplement plus, et la tolérance signifiait alors que chacun est libre d'adopter les principes les plus adaptés aux fins qu'il s'est fixées. Selon l'une des formulations les plus célèbres du principe de tolérance: "Nous ne voulons pas poser des interdits, mais fixer des conventions. [...] En logique, il n'y a pas de morale. Chacun est libre de construire sa logique, c'est-à-dire sa forme de langage, comme il l'entend. S'il veut discuter avec nous, il doit seulement indiquer clairement comment il entend procéder, et fournir des règles syntaxiques plutôt que des arguments philosophiques ${ }^{8} \gg$.

Avant d'examiner plus avant la position pluraliste de Carnap et de commenter ces déclarations, voyons ce qui le conduisit à cette position radicale, en commençant par donner quelques éléments du contexte dans lequel il élabora son projet philosophique dans les années 1920, avant le tournant pluraliste. L'une des objections qui sont parfois adressées à Carnap est en effet qu'il n'aurait pas donné de raison convaincante de renoncer à la position la plus commune (selon laquelle il y a une logique : la logique) pour adopter le pluralisme?.

7. Sur cette question, cf. S. Awodey et A. Carus, «From Wittgenstein's Prison to the Boundless Ocean : Carnap's Dream of Logical Syntax », in P. Wagner (éd.), Carnap's Logical Syntax of Language, Basingstoke, Palgrave Macmillan, 2009.

8. R. Carnap, Logische Syntax der Sprache [Syntaxe logique du langage], Vienne, Springer, 1934, p. 44-45. Â l'époque où Carnap écrit ces lignes, il pense encore que la logique pourra se réduire à la syntaxe, ce qui explique la mention des « règles syntaxiques » dans la citation. Il s'agit d'un point qui, pour notre propos, n'est pas essentiel. Quelques années plus tard, l'approche syntaxique sera complétée par une approche sémantique.

9. C'est l'une des objections formulées par P. Köllner, op. cit. 


\section{Versions de l'universalisme : Frege, Russell et Wittgenstein}

La formation logique de Carnap ne le prédisposait nullement à une prise de position en faveur du pluralisme. Dans l'un des passages de son autobiographie intellectuelle où il est question des années 1920, Carnap cite Frege, Russell et Wittgenstein comme trois auteurs qui eurent une influence décisive sur sa pensée, ce qui vaut tout particulièrement pour ce qui concerne la logique et la philosophie de la logique ${ }^{10}$. Or ces trois auteurs comptent parmi les principaux représentants de l'universalisme qui, à l'opposé de toute forme de pluralisme, pose que la logique peut être comprise comme une langue dont les règles ont une valeur universelle.

Frege appartient à une tradition selon laquelle la logique donne les conditions les plus générales du discours doué de sens ainsi que les règles les plus générales du raisonnement, applicables à tout domaine d'investigation dans lequel une méthode rationnelle est adoptée en vue d'atteindre de quelconques connaissances. Selon cette conception, ne pas respecter les règles de la logique, ce n'est pas commettre des erreurs de raisonnement ; c'est plutôt, en fonction des règles qui sont enfreintes, soit ne pas raisonner $\mathrm{du}$ tout, soit, plus radicalement, proférer des sons dépourvus de toute signification, et donc ne rien dire. Selon Frege, il n'y a donc aucun sens à chercher à fonder les principes de la logique dans une autre discipline car tout raisonnement et toute discipline qui vise une connaissance, tout énoncé doué de sens et toute inférence déductive valide présupposent eux-mêmes certaines règles logiques qui ne sauraient être fondées, ni dans une ontologie, ni dans une théorie de la connaissance ou une psychologie : «Je tiens comme un signe certain d'erreur que la logique ait besoin de la métaphysique et de la psychologie, sciences qui requièrent elles-mêmes les principes logiques ${ }^{11} \gg$. Quant au pouvoir d'autojustification de la logique, on comprend qu'il rencontre nécessairement une limite : « À la question de savoir pourquoi et de quel droit nous reconnaissons une loi de la logique comme vraie, la logique ne peut répondre qu'en la ramenant à une autre loi de la logique. Lorsque cela n'est pas possible, la logique ne peut apporter aucune réponse $\mathrm{e}^{12} \gg$. La logique est donc conçue comme ayant valeur de science première, qui délimite en toute clarté le domaine de l'investigation rationnelle en général. Sur ce point précis, Carnap est dans la continuité directe de la pensée de Frege, dont il avait suivi les cours à Iéna dans les années qui précèdent la Première Guerre mondiale : les règles logiques ne sont en aucune façon fondées sur une ontologie ou une théorie de la connaissance antérieure. Bien au contraire, aucune investigation visant la vérité ne saurait être développée sans l'établissement préalable des règles

10. R. Carnap, «Intellectual Autobiography», in P. A. Schilpp (éd.), The Philosophy of Rudolf Carnap, Chicago, Open Court, 1963, p. 25.

11. G. Frege, Grundgesetze der Arithmetik [Les lois fondamentales de l'arithmétique], vol. 1, 1893, Hildesheim, Olms, 1962, p. IX.

12. G. Frege, op. cit, p. XVII. 
logiques du langage dans lequel des contenus de connaissance peuvent être exprimés.

L'idée que le respect de certaines lois logiques universelles conditionne le sens de nos énoncés n'est, comme telle, pas directement liée à l'état de la logique à l'époque à laquelle Carnap mène ses recherches. Elle vaudrait également, par exemple, pour la logique générale pure au sens kantien du terme. Mais dans les années 1920, le recours à la logique comme discipline première, cadre nécessaire de tout discours doué de sens et de toute recherche de la vérité, se comprend surtout au regard de l'extraordinaire pouvoir d'analyse et d'expression que possède ce que Carnap appelle alors la «nouvelle logique », dont les trois volumes des Principia Mathematica de Russell et Whitehead donnent, à cette époque, à la fois l'exposé le plus complet et l'application la plus spectaculaire, le but de cet ouvrage étant de permettre la reconstruction de toutes les mathématiques dans la logique. La nouvelle logique est, à cet égard, d'une richesse et d'une fécondité très supérieures à celles de l'ancienne logique, dont le modèle d'analyse remontait à l'Antiquité, et dont les lois tenaient en quelques principes généraux (principes du tiers exclu, d'identité, de non-contradiction, etc.). Frege avait montré de manière convaincante les limites de l'analyse du jugement comme lien entre un prédicat et un sujet et Carnap put trouver une confirmation des différences essentielles qui séparent l'ancienne et la nouvelle logique lorsqu'il se livra à l'étude des Principia Mathematica, vers 1919. Peu de temps après, s'inspirant des idées que Russell avaient exposées dans une série de conférences publiées en 1914 sous le titre Our Knowledge of the External World ${ }^{13}$, dont il prit connaissance en 1921, il eut l'idée d'appliquer la nouvelle logique non seulement aux mathématiques mais à la science tout entière ainsi qu'à l'analyse et au traitement des problèmes philosophiques. Il conçut, en particulier, le projet d'une reconstruction rationnelle unifiée du système de tous les concepts en usage dans les sciences, projet mis en ouvre dans La Construction logique du monde ${ }^{14}$, ouvrage auquel Carnap travailla dès 1922 et dont une première version fut achevée en 1925. À cette époque, et jusqu'au début des années 1930, le cadre logique dans lequel Carnap élaborait ce projet était encore celui de l'universalisme.

La troisième influence majeure est celle du Tractatus logicophilosophicus $^{15}$, dont de larges extraits étaient lus et discutés par les membres du Cercle de Vienne au milieu des années 1920. Carnap y découvrit l'idée, qu'il retiendra constamment par la suite, selon laquelle les énoncés de la logique, étant vrais quel que soit l'état des choses dans le monde, ne dépendent pas des faits contingents : ils ne disent rien du monde

13. B. Russell, La Méthode scientifique en philosophie et notre connaissance du monde extérieur (1914), trad. Ph. Devaux, Paris, Payot, 1971 ; Payot et Rivages, 2002.

14. R. Carnap, La Construction logique du monde (1928), trad. Th. Rivain, Paris, Vrin, 2002. 15. L. Wittgenstein, Tractatus Logico-Philosophicus (1922), trad. G.-G. Granger, Paris, Gallimard, 1993. 
et sont donc vides de tout contenu factuel. Pour Wittgenstein (à l'époque du Tractatus), les propositions logiques ne «traitent » de rien, elles « décrivent l'échafaudage du monde », ou plutôt elles le «figurent » (aphorisme 6.124 du Tractatus). Dans cette perspective, «la logique n'est point une théorie, mais une image qui reflète le monde. La logique est transcendantale» (aphorisme 6.13). Comparées à celles de Frege ou de Russell, ces idées sur le caractère tautologique des propositions de la logique font de Wittgenstein un penseur extrêmement original, qui n'en demeure pas moins, dans l'ouvrage de 1922, encore très attaché à une conception universaliste. Aussi pouvait-il également y écrire que «nous ne pouvons rien penser d'illogique, parce que nous devrions alors penser illogiquement» (aphorisme 3.03). La logique, loin d'être pensée comme un ensemble de règles ou de lois que l'on pourrait imaginer modifier ou transformer par voie de décision ou de stipulation, est, ici aussi, plutôt à concevoir comme l'ensemble des règles qui, implicitement, définissent les conditions de possibilité de tout discours doué de sens.

Dans les années 1920, les influences conjuguées de Frege, Russell et Wittgenstein conduisent assez naturellement Carnap à se placer dans la perspective d'une conception universaliste de la logique ${ }^{16}$, mais également, dans le même temps, à défendre un curieux syncrétisme selon lequel, premièrement, la logique est une science première qui ne saurait être fondée dans aucune autre discipline de base (Frege), deuxièmement les mathématiques peuvent être reconstruites sur la base de la logique, dont elles constituent une simple branche (Russell), et à la fois, troisièmement, les propositions de la logique sont des tautologies, c'est-à-dire des propositions vides de sens et dépourvues de tout contenu factuel (Wittgenstein) ${ }^{1 \dagger}$.

\section{Philosophie scientifique et rationalisme logique}

Pour comprendre ce qui conduisit Carnap, au début des années trente, à renoncer à l'universalisme en faveur d'une forme de pluralisme logique et à

16. Sur laquelle il n'avait, bien entendu, pas la perspective et le recul que nous pouvons avoir lorsque nous l'opposons, rétrospectivement, au pluralisme logique ou à l'approche en termes de théorie des modèles. Carnap, du reste, n'utilise pas le mot «universalisme », dont l'introduction, dans ce contexte, est beaucoup plus tardive. Sur l'idée même d'universalisme logique, le locus classicus est J. Van Heijenoort, «Logic as calculus and logic as language », Synthese, 17, 1967, p. 324-330. Sur la même question, un autre classique (dans lequel, curieusement, l'article de Van Heijenoort n'est pas cité) est W. Goldfarb, «Logic in the Twenties: The Nature of the Quantifier », The Journal of Symbolic Logic, 44, 1979, p. 351368. Cf. également F. Rivenc, Recherches sur l'universalisme logique: Russell et Carnap, Paris, Payot, 1993.

17. Carnap expose ses idées et l'état de ses recherches en logique et sur les fondements des mathématiques dans une série de textes parus entre 1927 et 1931. Cf. par exemple «L'ancienne et la nouvelle logique» (1930), trad. Ch. Bonnet, in P. Wagner (éd.), De l'universalisme au pluralisme logique, Paris, Vrin, à paraître ; ou encore, «La fondation logiciste des mathématiques » (1931), trad. F. Rivenc, in P. Wagner, op. cit. 
l'adoption du principe de tolérance, il ne suffit pas d'apercevoir les limites et les apories du syncrétisme logique qu'il avait cherché à élaborer au cours des années vingt dans le cadre d'une réflexion sur le problème du fondement des mathématiques; il faut également saisir comment ses travaux sur la «nouvelle logique » sont articulés à un projet philosophique beaucoup plus général, dont la constitution de tous les concepts en un système unifié est l'un des principaux aspects. La signification du tournant pluraliste n'est nullement limitée à une question de philosophie de la logique : elle s'inscrit dans un mouvement philosophique, et même extra-philosophique, de grande ampleur, au sein duquel Carnap occupe une position singulière.

Dans la préface de La Construction logique du monde, Carnap fait explicitement le lien entre le développement de la nouvelle logique et l'idée d'un changement de méthode en philosophie. Aux systèmes traditionnels fondés sur l'intuition personnelle d'un auteur, dont l'histoire de la philosophie offre de nombreux exemples, il oppose une conception scientifique de la philosophie comme entreprise collective de recherche :

Historiquement, il est compréhensible que la nouvelle logique n'ait d'abord intéressé que le cercle étroit des mathématiciens et des logiciens. Seul un petit nombre a pressenti sa remarquable importance pour toute la philosophie et cet élargissement de son champ d'utilisation ne fait que commencer. Si la philosophie est disposée à suivre le chemin de la science (au sens strict), elle ne saurait renoncer à ce moyen particulièrement efficace d'élucidation des concepts et de clarification de l'état de ses questions. [...] La nouvelle manière de philosopher est née d'un contact étroit avec le travail des scientifiques, notamment en mathématique et en physique. En conséquence, le philosophe s'attache à adopter l'attitude fondamentale du chercheur scientifique faite de rigueur et de sens des responsabilités tandis que celle du philosophe de l'ancienne école l'apparente davantage au poète. Ce nouveau comportement ne change pas seulement le style de penser mais aussi la manière de poser les tâches à accomplir. Un individu ne se lance plus dans l'entreprise téméraire de construire l'édifice complet de la philosophie. Chacun au contraire travaille à sa place déterminée au sein de l'ensemble de la science qui est une $e^{18}$.

Le projet philosophique de Carnap était donc non seulement de travailler à l'établissement et à la clarification des fondements d'une logique universelle mais également d'en développer les applications philosophiques et d'en tirer les moyens d'une réforme de la méthode philosophique : la clarification des concepts par analyse logique se conçoit comme une entreprise collective dont La Construction logique du monde offre une première ébauche.

Ici, il importe de souligner d'une part que les motivations de ce projet étaient loin d'être purement théoriques et d'autre part que Carnap le

18. R. Carnap, La Construction logique du monde (1928), Paris, Vrin, 2002, p. 53-54 (Traduction légèrement modifiée). 
concevait explicitement comme relevant d'un mouvement de pensée qui dépassait le champ proprement philosophique. Inspiré par une tradition qui remontait à la philosophie des Lumières, le projet carnapien s'inscrivait dans un combat contre différentes formes d'irrationalisme présentes dans l'univers intellectuel de l'époque, en Allemagne notamment, et la clarification conceptuelle par analyse logique du langage qui en constituait la partie théorique la plus visible était orientée par des idéaux d'ordre politique et social, ce dont témoigne de manière parfaitement claire à la fois la biographie du jeune Carnap ${ }^{19}$ et le fameux Manifeste du Cercle de Vienne, à la rédaction duquel on sait qu'il contribua, qui revendique explicitement à la fois l'héritage des Lumières et une «conception scientifique du monde $»^{20}$. Carnap évoque également cette dimension pratique du projet philosophique qui anime La Construction logique du monde, ainsi que l'accord du projet avec l'esprit du temps, dans la préface de l'ouvrage :

Nous ne pouvons pas nous dissimuler que précisément aujourd'hui, dans les domaines métaphysique et religieux, les courants qui s'opposent à une telle attitude exercent de nouveau une puissante influence. Qu'est-ce qui nous permet malgré tout d'espérer que notre appel en faveur de la clarté et pour une science débarrassée de métaphysique l'emporte, sinon la conscience, ou pour le dire plus modestement, la croyance que ces forces contraires appartiennent au passé ? Nous ressentons une intime parenté entre l'attitude sous-jacente à notre travail philosophique et cette attitude spirituelle qui travaille actuellement des aspects de la vie tout à fait différents ; nous la percevons dans des courants artistiques, en architecture notamment, et dans les mouvements qui s'attachent à donner à la vie humaine une forme rationnelle : à la vie personnelle et collective, à l'éducation, en somme à ce qui règle la vie extérieure. C'est partout que nous remarquons ici cette même attitude fondamentale, un même style de penser et d'agir. Cet esprit introduit la clarté dans tous les domaines, tout en reconnaissant la complexité de la vie qui n'est jamais totalement pénétrable ; il mène à la fois au soin dans la configuration du détail et à la saisie des grandes lignes de l'ensemble ; il concilie la solidarité entre les hommes et le libre épanouissement de l'individu. Cet esprit appartient à l'avenir, telle est la croyance qui porte notre travail ${ }^{21}$.

19. Cf. les premiers chapitres d'A. W. Carus, Carnap and Twentieth-Century Thought. Explication as Enlightenment, Cambridge, Cambridge University Press, 2007. Cf. aussi J. Bouveresse, « Rudolf Carnap and the Legacy of Aufklärung », in P. Wagner (éd.), Carnap's Ideal of Explication and Naturalism, Basingstoke, Palgrave Macmillan, à paraître.

20. Cet opuscule parut sans nom d'auteur (seule la brève préface était signée) sous le titre : «La conception scientifique du monde. Le Cercle de Vienne» (1929), trad. A. Soulez et al., in A. Soulez (éd.), Manifeste du Cercle de Vienne et autres écrits, Paris, Vrin, 2010. On y lit par exemple, p.106 : «Tout au long de la seconde moitié du XIX ${ }^{\mathrm{e}}$ siècle, le libéralisme était la tendance politique dominante à Vienne. Les sources de son univers intellectuel sont les Lumières, l'empirisme, l'utilitarisme et le libre-échangisme anglais. [...] Cet esprit des Lumières plaçait Vienne à la pointe de l'éducation populaire scientifiquement orientée ».

21. R. Carnap, op. cit., p. 55. 
Aux antipodes du modèle traditionnel d'un système qui reflète l'intuition singulière de son auteur, le projet philosophique de Carnap se réfère explicitement, eu égard à sa méthode, à un mode de travail collaboratif qui vise le type d'intelligibilité et de rationalité que l'on peut trouver dans une certaine pratique scientifique. Mais il se comprend également, on le voit, comme une contribution à un mouvement collectif, pensé dans la continuité d'une tradition philosophique qui remonte au combat des Lumières contre différentes formes d'irrationalisme et d'intolérance, y compris dans sa dimension pratique. Pour Carnap comme pour cette partie du mouvement qui prendra ultérieurement le nom d' "empirisme logique», un tel combat prenait typiquement la forme d'une lutte contre la métaphysique - en particulier dans sa version dite «théologique »-, lutte dont l'analyse logique du langage constituait l'un des principaux instruments.

Dans ce contexte, on comprend l'importance cruciale des recherches qui visaient à clarifier le statut épistémologique de la "nouvelle logique », car celle-ci était investie d'une fonction qui en faisait la clef d'un projet philosophique ambitieux. Beaucoup trop ambitieux, en réalité, car la question de l'essence de la logique se révéla bien plus complexe que ce qu'avaient d'abord imaginé les auteurs du programme esquissé dans le Manifeste du Cercle de Vienne ${ }^{22}$. La logique, censée être constituée de pures tautologies (au sens d'énoncés qui ne nous apprennent rien sur l'état des choses dans le monde), devait, selon ce programme, non seulement servir de base à la reconstruction rationnelle de tous les concepts en usage dans les sciences du réel - c'est le programme de La Construction logique $d u$ monde-, mais également permettre d'établir que les énoncés de la métaphysique sont dépourvus de toute signification cognitive ${ }^{23}$ et que les mathématiques peuvent être reconstruites comme une «branche» de la logique ${ }^{24}$.

La plupart des représentants du mouvement pour une conception scientifique du monde comprirent assez rapidement que bien des objectifs de l'ambitieux programme de la fin des années 1920 ne pouvaient en réalité être atteints, du moins tels qu'ils avaient été initialement conçus. Carnap, pour sa part, ne renonça cependant pas à l'orientation profonde de son projet philosophique, qui impliquait une critique de la métaphysique, un traitement uniforme des propositions de la logique et des mathématiques et une reconstruction rationnelle des concepts de la science du réel en un système

22. Une version similaire du programme (dans laquelle on peut observer le même excès d'optimisme) est exposée dans A. Blumberg et H. Feigl, "Le positivisme logique. Un nouveau courant dans la philosophie européenne » (1931), trad. P. Wagner, in Ch. Bonnet et P. Wagner (éds.), L'Âge d'or de l'empirisme logique, Paris, Gallimard, 2006, p. 135-159.

23. Cf. R. Carnap, «Le dépassement de la métaphysique par l'analyse logique du langage» (1931), trad. B. Cassin et al., in A. Soulez (éd.), Manifeste du Cercle de Vienne et autres écrits, Paris, Vrin, 2010, p. 149-171.

24. R. Carnap, «Les mathématiques comme branche de la logique» (1930), trad. D. ChapuisSchmitz, in P. Wagner (dir.), De l'universalisme au pluralisme logique, Paris, Vrin, à paraître. 
unifié. Mais au début des années 1930, il comprit que la réalisation de ce programme exigeait que certaines de ses conceptions fondamentales soient réformées. L'adoption du pluralisme logique et du principe de tolérance fut certainement, à cet égard, la décision de réforme la plus radicale ${ }^{25}$. Dans le même temps, cette conception nouvelle de la logique rendait également possible une nouvelle conception de l'a priori, du rapport à la métaphysique, et de la méthode philosophique.

\section{Le choix du pluralisme logique et de la tolérance}

Renoncer à l'universalisme ne fut pas la décision d'un seul jour et l'histoire complexe de ce tournant majeur de la pensée de Carnap a fait l'objet d'études historiques minutieuses dont nous ne reprenons pas ici le détail $^{26}$. Pour nous en tenir à l'essentiel, disons que l'idée fondamentale était, premièrement, qu'un langage est défini par des règles formelles (règles de formation qui définissent quelles suites de signes ont valeur d'énoncés, et règles de transformation qui définissent à quelles conditions un énoncé est conséquence d'un ensemble d'énoncés); deuxièmement, que des choix différents pour ces règles - et donc pour une variété de langages possibles peuvent être analysés, étudiés et discutés dans un métalangage (alors que les représentants de la philosophie universaliste ne concevaient évidemment pas la possibilité d'une distinction principielle entre plusieurs langages et n'avaient donc pas théorisé l'idée d'un métalangage); enfin et surtout, troisièmement, que le choix d'adopter tel ensemble de règles (c'est-à-dire tel langage particulier, mais également - parce que les règles définissent un concept de conséquence - telle logique particulière) ne saurait recevoir aucune justification ontologique ou épistémologique, parce qu'il n'y a rien par rapport à quoi ces règles puissent être dites ni correctes ou incorrectes, ni vraies ou fausses; aussi la décision, sur ce point, est-elle purement pragmatique, au sens où ce qui la justifie est de l'ordre de la convenance, de la simplicité ou de l'opportunité, non de la vérité.

Remarquons que si l'adoption d'un cadre linguistique de référence (qui comprend un ensemble de règles logiques) ne saurait être dite correcte ou incorrecte, si, en d'autres termes, il s'agit d'une décision pratique qui n'est pas fondée sur une connaissance théorique, le principe de tolérance lui-même ne doit pas davantage être interprété comme une thèse qui serait susceptible d'être prouvée ou justifiée, ni même qualifiée de vraie ou de fausse. Il n'est certes pas impossible d'envisager une discussion sur la question de savoir s'il existe réellement une ou plusieurs logiques (à distinguer de la question

25. Paradoxalement, elle fut également, pendant longtemps, la moins remarquée et la moins commentée. De là de fréquents contresens sur le sens de sa pensée, chez un grand nombre de critiques et de commentateurs.

26. Cf. les références indiquées ci-dessus dans les notes 7 et 19 (p. 100 et 105). 
de savoir s'il existe plusieurs langages définis par des règles, y compris des règles de transformation qui définissent une relation de conséquence), mais la décision prise par Carnap est de ne pas s'engager dans une forme de discussion qui, pour régler cette question, ferait référence à une ontologie, une épistémologie ou une psychologie, précisément pour les raisons invoquées par Frege : tout raisonnement qui se rapporterait à l'une de ces disciplines présuppose un cadre logique, c'est-à-dire justement la définition des règles logiques que l'on est prêt à suivre dans le raisonnement. Le principe de tolérance doit donc être compris comme une suggestion, ou une recommandation: celle, premièrement, de ne pas chercher à justifier les principes de la logique sur une ontologie ou une théorie de la connaissance et, deuxièmement, de remplacer toute justification des principes logiques par une exposition des règles que l'on souhaite adopter comme définissant un cadre logique de référence. La position adoptée par Carnap est à la fois pluraliste et anti-fondationaliste, comme le montre l'exposition du principe de tolérance qu'on peut lire dans l'Avant-propos de La Syntaxe logique du langage :

[Dans ce livre], on défendra l'idée que nous avons à tout point de vue une liberté complète à l'égard des formes de langage ; qu'aussi bien les règles de construction pour les propositions que les règles de transformation (ces dernières sont habituellement désignées sous les noms de "postulats » et de «règles d'inférence ») peuvent être choisies de façon tout à fait arbitraire. [...] L'utilisation de cette méthode a en outre pour effet de faire disparaître les divergences de position sur le problème des fondements des mathématiques. Car le langage, pour ce qui est de sa forme mathématique, peut être construit conformément aux préférences de l'une quelconque des tendances représentées; de sorte qu'il ne se pose absolument aucune question de justification, mais uniquement le problème des conséquences syntaxiques auxquelles conduit l'un ou l'autre des choix. [...] Les premières tentatives qui ont été effectuées pour détacher le vaisseau logique de la terra firma des formes classiques étaient assurément audacieuses, si on les considère du point de vue historique. Mais elles ont été entravées par le fait que l'on s'efforçait de réaliser quelque chose de «correct». Maintenant, en revanche, cet obstacle a été surmonté, et devant nous s'étend l'océan immense des possibilités illimitées ${ }^{27}$.

On pourrait supposer que le pluralisme logique, tel que le comprend Carnap dans ce texte, se justifie par une conviction philosophique préalablement acceptée, selon laquelle seuls sont doués de signification les énoncés qui sont susceptibles d'être vérifiés (ou confirmés, ou testés) dans l'expérience. En d'autres termes, la thèse du pluralisme pourrait être conçue comme implicitement fondée sur une théorie vérificationniste de la signification qui exprimerait des convictions empiristes. Selon ce point de vue, si l'adoption d'un cadre logique doit être conçue comme une question

27. R. Carnap, Logische Syntax der Sprache, Vienne, Springer, 1934, p. v-vi. 
de pure décision ou de stipulation, c'est parce que la correction d'une règle de logique n'est pas susceptible d'être vérifiée dans l'expérience, et qu'en cherchant à donner une justification épistémologique à l'adoption d'un ensemble de règles logiques, on verserait nécessairement dans une des formes de métaphysique que Carnap et les empiristes logiques refusaient précisément de reconnaître comme douées de sens.

Une telle interprétation ne rend cependant pas compte de la radicalité du tournant opéré par Carnap, car on voit mal comment le principe du vérificationnisme, qui n'est pas lui-même susceptible d'être vérifié dans l'expérience, pourrait à son tour être conçu comme une thèse, vraie ou fausse. Selon une critique bien connue, en effet, si le principe du vérificationnisme était une thèse, il devrait alors lui-même être rejeté comme dépourvu de signification en vertu du principe qu'il énonce. Le principe du vérificationnisme doit donc plutôt être lui-même compris comme une proposition (au sens d'une suggestion) que l'on peut recommander d'adopter, et il ne saurait, en conséquence, être conçu comme une justification de la vérité du pluralisme logique ${ }^{28}$.

L'idée fondamentale de Carnap est de renoncer à l'idée de correction en logique et de remplacer l'éventuelle fondation de la logique par une stipulation. En choisissant d'adopter cette position, Carnap reste fidèle à l'idée frégéenne selon laquelle aucune discipline ne peut être établie en dehors d'un cadre logique premier; il renonce, en revanche, à l'universalisme et donc à l'idée qu'il y aurait une seule logique (et, fondamentalement, un seul langage ${ }^{29}$ ).

Le dernier texte cité fait également apparaître l'une des motivations de Carnap pour l'adoption du pluralisme logique et du principe de tolérance : «faire disparaître les divergences de position sur le problème des fondements des mathématiques ». À strictement parler, les divergences de position demeurent, même dans le cadre que propose Carnap, celui d'une exposition systématique des règles logiques que l'on souhaite adopter et de leur discussion dans un métalangage. Mais il est clair que ces divergences n'ont plus alors la même signification : la comparaison des conséquences qui résultent de l'adoption de tel système de règles ou de tel autre, de tel langage ou de telle structure logique plutôt qu'un ou une autre, remplace les discussions philosophiques sur des questions de fondement. Cette position illustre bien l'orientation radicalement déflationniste que prend la pensée de Carnap, qui suggère de laisser à l'écart les discussions philosophiques

28. Sur cette question, cf. Th. Ricketts, «Carnap's Principle of Tolerance, Empiricism, and Conventionalism », in P. Clark et B. Hale (éds.), Reading Putnam, Oxford, Blackwell, 1994, p. 176-200.

29. Sur le sens de « un seul langage », dans ce contexte, cf. l'aphorisme 3.343 du Tractatus de Wittgenstein : «Les définitions sont des règles de traduction d'une langue dans une autre. Tout symbolisme correct doit pouvoir être traduit dans tout autre au moyen de telles règles : c'est cela qu'ils ont tous en commun ». Le pluralisme logique renonce à l'idée même de « symbolisme correct ». 
infructueuses ou stériles dont font typiquement partie, selon lui, les débats sur le fondement des mathématiques tels qu'ils avaient cours dans les années 1920, entre l'intuitionnisme, le formalisme et le logicisme. Ici encore, Carnap n'a pas l'ambition de prouver qu'il n'y a pas de fondement aux règles - y compris logiques - qui définissent un langage, ou le langage dont nous faisons usage ; il propose ou suggère plutôt de renoncer à chercher à résoudre le problème du fondement de cette manière, et tel est le sens de l'adoption du principe de tolérance.

\section{Tolérance et empirisme}

En renonçant à l'universalisme logique au profit du pluralisme et de la tolérance, Carnap ne renonçait pas au projet philosophique général dont nous avons indiqué quelques éléments. Mais une révision aussi radicale de sa philosophie de la logique ne pouvait rester sans conséquence pour ce projet, car la logique en constituait le principal outil méthodologique. Le tournant pluraliste entraînait notamment une révision des modalités de la critique de la métaphysique et de l'analyse des propositions logico-mathématiques comme vides de contenu, deux des piliers de l'empirisme carnapien.

En 1932 paraissait le (trop) fameux article sur «Le dépassement de la métaphysique par l'analyse logique du langage $»^{30}$, l'un des rares écrits de Carnap dont une traduction française avait été publiée au siècle dernier. Carnap tentait d'y montrer que les énoncés de la métaphysique n'ont pas davantage de sens qu'une suite de mots comme "César est un nombre premier » et se plaisait à comparer les métaphysiciens à « des musiciens sans talent musical ${ }^{31}$. Ce texte, qui fut rédigé avant le tournant pluraliste, expose une version de la critique de la métaphysique que Carnap n'a en fait défendue que pendant une période assez brève, et il est très regrettable que de telles formulations continuent, aujourd'hui encore, à être conçues comme offrant une représentation adéquate de la pensée de leur auteur dans son ensemble ${ }^{32}$. Carnap semble effectivement avoir nourri, pendant un temps, l'espoir que l'analyse logique (fondée sur la logique universelle) des énoncés permette, premièrement, d'établir que les énoncés de la métaphysique sont dépourvus de sens et, deuxièmement, de caractériser le contenu de signification des énoncés des sciences du réel. Si l'on suppose qu'il y a un langage logique (dans lequel les énoncés doués de sens des différentes langues peuvent être formulés) et donc une logique universelle, on peut bien concevoir le projet d'une distinction entre trois catégories de

30. R. Carnap, op. cit., (cf. supra, note 23, p. 106).

31. Op. cit., p. 169.

32. La diffusion de ce texte, et de quelques autres, a largement contribué à forger la réputation détestable et parfaitement imméritée qui reste encore attachée au nom de son auteur: celle d'une pensée sectaire, intolérante et profondément antiphilosophique. 
formulations qui, dans le langage usuel, prétendent au titre d'énoncé : 1) celles dont la traduction dans le langage logique révèle qu'il s'agit en réalité de pseudo-énoncés dépourvus de signification ; 2) celles qui se réduisent à des lois logiques ; 3) celles dont l'analyse met en évidence le contenu exact de leur signification. En prenant le tournant pluraliste, Carnap renonce à trouver dans la logique censée être universelle le principe d'une séparation rigoureuse entre propositions métaphysiques, propositions logicomathématiques et propositions des sciences de la nature, conçu comme recouvrant la tripartition précédente.

Pour autant, il ne renonce ni à la critique de la métaphysique par analyse logique du langage, ni à l'idée que les énoncés des sciences formelles (logique et mathématiques) ont pour caractère commun l'absence de tout contenu de signification. Mais après l'adoption du principe de tolérance, ces idées prennent un sens singulièrement différent puisque Carnap suggère alors de remplacer la pratique de la philosophie au sens traditionnel par la logique de la science ${ }^{33}$, et donc par l'analyse des langages dans lesquels une reconstruction systématique des énoncés qui expriment une forme de connaissance est possible. L'empirisme, alors défini par une forme de vérificationnisme (mais dans lequel l'exigence de confirmabilité ou de testabilité remplace celle de vérifiabilité), n'est plus conçu comme une thèse ou une affirmation mais comme une exigence ou une recommandation:

Il me semble préférable de formuler le principe de l'empirisme non pas sous la forme d'une affirmation - «toute connaissance est empirique » ou « tous les énoncés synthétiques que nous pouvons connaître sont fondés sur (ou liés à) des expériences » ou d'autres formulations du même genre - mais plutôt sous la forme d'une proposition ou d'une exigence. En tant qu'empiristes, nous requerrons du langage de la science qu'il soit restreint d'une certaine manière ; nous requerrons que les prédicats descriptifs, et donc les énoncés synthétiques, ne soient pas admis à moins d'avoir quelque lien avec des observations possibles, un lien qui doit être caractérisé de manière adéquate. Il me semble qu'une telle formulation apportera une plus grande clarté, tant pour la poursuite des débats entre empiristes et anti-empiristes que pour les réflexions des empiristes $^{34}$.

On reconnaît dans l'article dont est extrait ce passage plusieurs caractères de l'orientation que prend la pensée de Carnap à partir du tournant des années 1930. Premièrement, un déflationnisme philosophique qui consiste à remplacer l'activité de théorisation des questions philosophiques, telle qu'elle est traditionnellement conçue, par une recherche sur les formes de langage possibles, pratiquée dans un métalangage (typiquement, au lieu

33. «La logique de la science prend la place de l'enchevêtrement inextricable de problèmes que l'on désigne du nom de philosophie. », R. Carnap, Logische Syntax der Sprache, Vienne, Springer, 1934, p. 205.

34. R. Carnap, Testabilité et signification, (1936-1937), trad. Y. Benétreau-Dupin, Paris, Vrin, à paraître, $\S 27$. 
de s'interroger pour savoir si toute connaissance est empirique, rechercher quelles sont les formes de langage dans lesquels les prédicats descriptifs ne sont admis qu'à condition d'entretenir certaines relations - sur lesquelles Carnap donne des précisions - avec des observations possibles, et analyser les conséquences de l'adoption de ces formes de langage), où l'on reconnaît un exemple caractéristique du tournant linguistique en philosophie. Deuxièmement, l'application du pluralisme et de la tolérance, qui valent présentement non seulement pour les règles logiques mais également pour les formes de langage en un sens plus général (notamment le choix des prédicats descriptifs). Enfin, troisièmement, l'idée selon laquelle le choix du langage est une question de décision pratique, non de connaissance théorique. Voici comment Carnap expose ce troisième point :

Mais même si $\mathrm{L}$ devait être un langage adéquat pour toute la science, nombreux sont ceux - dont je fais partie - qui ne souhaiteraient pas que L contienne un énoncé correspondant à tout énoncé habituellement considéré comme un énoncé correct du français et employé par les gens instruits. Nous ne souhaiterions pas, par exemple, avoir les énoncés qui correspondent à un grand nombre, ou à la plupart, des énoncés qu'on trouve dans les livres des métaphysiciens. Ou, pour donner un exemple non-métaphysique, les membres de notre Cercle ne souhaitaient pas autrefois inclure dans notre langage de la science un énoncé correspondant à l'énoncé français S1: «Cette pierre pense maintenant à Vienne». [...] Notre erreur fut simplement de ne pas avoir reconnu que la question était une question de décision concernant la forme du langage ; nous avons exprimé par conséquent notre conception sous la forme d'une affirmation - ce qui est courant parmi les philosophes - plutôt que sous la forme d'une proposition ${ }^{35}$.

Le point essentiel est que le problème philosophique initial est remplacé par la question du choix d'un certain langage, qui relève d'une décision. Et la suggestion de Carnap est alors d'adopter une forme de langage dans laquelle on ne puisse former ni des énoncés correspondant à ceux de la métaphysique ni des énoncés qui enfreignent certaines règles de la logique du langage usuel comme « Cette pierre pense maintenant à Vienne».

De manière similaire, alors que dans les années 1920, Carnap défendait la thèse selon laquelle les mathématiques sont une branche de la logique et qu'elles sont dépourvues de tout contenu cognitif (parce qu'il n'existe aucun domaine d'objets mathématiques qu'elles nous feraient connaître), dans les années 1930, après l'adoption du principe de tolérance, il ne renonce pas à l'idée que les énoncés mathématiques sont vides de contenu (ces énoncés sont analytiques), mais cette idée prend alors la forme d'une suggestion relative au choix d'un langage. La proposition empiriste (du moins celle de l'empirisme carnapien) est donc d'adopter un langage dans lequel les énoncés qui correspondent aux énoncés logico-mathématiques du langage usuel peuvent être reconstruits comme des énoncés analytiques. On

35. R. Carnap, ibid. Les italiques sont ajoutés. 
reconnaît, ici encore, certains caractères de la pensée de Carnap déjà soulignés: son déflationnisme philosophique (ce qui était une thèse sur l'essence des mathématiques est remplacé par l'analyse d'un langage que l'on se propose de construire), le pluralisme (il existe plusieurs formes de langage, certains dans lesquels les mathématiques sont analytiques d'autres dans lesquels elles ne le sont pas), et la tolérance (le choix de telle forme de langage plutôt qu'une autre relève d'une décision pratique qui, sans être arbitraire, n'est pas pour autant fondée sur une connaissance théorique ou sur une ontologie - l'essence des choses ou un en soi des objets mathématiques.

\section{Carnap nietzschéen ?}

Dans le chapitre de la Dialectique transcendantale dans lequel il traitait de l'antinomie de la raison pure, Kant choisissait de nommer «empirisme » la doctrine formée des quatre « antithèses » (selon lesquelles 1. le monde n'a de commencement ni dans le temps ni dans l'espace, 2. il n'existe rien de simple, 3. il n'y a pas de liberté, et 4. il n'existe aucun être absolument nécessaire) et montrait que si l'empirisme ainsi défini pouvait être compris comme «une maxime qui nous recommanderait la modération dans nos prétentions et la réserve dans nos assertions ${ }^{36} \gg$ (recommandation de ne pas s'enquérir de ce qui dépasse le champ des simples expériences possibles), cette même doctrine «devient [elle]-même dogmatique par rapport à nos idées (comme il arrive le plus souvent), et s'il nie avec assurance ce qui est au-dessus de la sphère de ses connaissances intuitives, [l'empirisme] tombe alors à son tour dans le vice d'immodestie ${ }^{37}$ ». S'il veut éviter de verser dans le dogmatisme, et de se retourner ainsi en son contraire, ce que Kant appelle ici «empirisme» doit donc, selon lui, prendre la forme d'une recommandation, non celle d'une affirmation. On peut dire que l'empirisme carnapien des années 1920, comme l'empirisme au sens que Kant donne à ce terme dans le chapitre sur l'antinomie de la raison pure, versait dans une forme de dogmatisme et dans le vice d'immodestie lorsqu'il se proposait d'établir que les énoncés de la métaphysique sont (en soi) dépourvus de signification et que les mathématiques sont, par essence, une branche de la logique. De même que l'empirisme conçu, chez Kant, comme une doctrine formée de thèses (en fait les quatre antithèses de «L'antinomie de la raison pure ») se retourne en quelque sorte contre lui-même dans une forme de dogmatisme, de même le principe du vérificationnisme, s'il est conçu comme une thèse qui définirait l'empirisme, se retourne contre lui-même en offrant un argument qui permet d'établir qu'il est, précisément en raison de ce qu'il énonce, dépourvu de signification.

36. E. Kant, Critique de la raison pure, AK III 327. Trad. A. J.-L. Delamarre et F. Marty, Paris, Gallimard, Pléiade, vol. 1, p. 1123.

37. Ibid. 
La réponse de Carnap à cette objection bien connue repose d'une part sur l'introduction du point de vue métalinguistique, et donc, de fait, sur la version carnapienne du tournant linguistique (la philosophie est remplacée par une analyse logique des langages possibles, menée dans un métalangage) et, d'autre part, sur le tournant pluraliste et le principe de tolérance, qui font du choix d'un cadre logico-linguistique particulier une question de stipulation. Or pluralisme et tolérance sont eux-mêmes adoptés en conséquence d'une décision, en sorte que dans sa réponse, Carnap a recours à une double décision ${ }^{38}$ : premièrement, l'adoption du pluralisme et de la tolérance; deuxièmement, dans le cadre du pluralisme et de la tolérance, l'adoption d'un (ou la recommandation d'adopter un) cadre linguistique dans lequel les énoncés de la logique et des mathématiques peuvent être reconstruits en tant qu'énoncés analytiques et dans lequel il n'est pas possible de reconstruire comme étant doués de sens les énoncés de la métaphysique. La seconde décision peut en fait être conçue comme un véritable programme de recherche auquel Carnap continua de travailler jusqu'à la fin de sa vie : montrer qu'il est possible de définir avec précision un langage dans lequel ces deux exigences relatives aux mathématiques et à la métaphysique peuvent effectivement être satisfaites, ainsi que d'autres exigences, caractéristiques de l'empirisme carnapien ${ }^{39}$.

Cette interprétation de sa pensée permet d'apporter une réponse aux objections de certains commentateurs qui ont cru pouvoir détecter une tension dans l'empirisme de Carnap, entre, d'une part, le principe de tolérance et, d'autre part, l'idée selon laquelle les énoncés de la logique et des mathématiques sont analytiques ${ }^{40}$. Elle n'est cependant pas sans soulever de nouvelles difficultés et objections, dont l'une concerne la distinction de principe entre décision pratique et connaissance théorique, sur laquelle sont fondés le pluralisme et la tolérance. Si la préférence affichée pour un langage dont les énoncés métaphysiques sont exclus et dans lequel

\footnotetext{
38. On notera au passage qu'il s'agit de la réponse de Carnap, non celle des empiristes logiques en général. La réponse de Moritz Schlick, par exemple, est radicalement différente. La question de l'empirisme fit l'objet de très vifs débats au sein du Cercle de Vienne, notamment entre Carnap, Schlick et Neurath, qui ne s'entendent pas sur la manière de concevoir un empirisme conséquent.
}

39. Les « décisions » prises par Carnap relativement aux mathématiques et à la métaphysique sont deux exemples, parmi d'autres, des caractères qui définissent son orientation philosophique empiriste. Pour centrales qu'elles puissent être pour lui, elles ne suffisent évidemment pas à définir complètement son projet philosophique.

40. Comment prôner le pluralisme logique et la tolérance, en effet, si celle-ci dépend de la possibilité de séparer sciences formelles et sciences du réel, et donc de l'adoption d'une forme de logicisme qui rend possible une telle séparation? Cette difficulté est discutée par M. Friedman dans «Tolerance and Analyticity in Carnap's Philosophy of Mathematics », in M. Friedman, Reconsidering Logical Positivism, Cambridge, Cambridge University Press, 1999. Plus récemment, M. Friedman a proposé une nouvelle résolution de cette tension (différente de celle que nous venons d'exposer), in «Tolerance, Intuition, and Empiricism», in P. Wagner (éd.), Carnap's Logical Syntax of Language, Basingstoke, Palgrave Macmillan, 2009. 
les mathématiques sont analytiques ne repose pas, fondamentalement, sur une connaissance théorique relative aux prétendus objets de la métaphysique et des mathématiques, mais sur une décision pratique qui, sans être arbitraire, n'a de justification que purement pragmatique, alors se pose la question de la rationalité d'une telle décision et de la valeur du type de justification dont elle est susceptible. Si l'analyticité des mathématiques et le rejet de la métaphysique ne sont plus fondés sur le recours à l'analyse logique au sens de la logique universelle, on est en effet en droit de s'interroger d'une part sur la valeur des analyses logiques qui reposent sur des règles stipulées et, d'autre part, sur la nature exacte des raisons «pragmatiques » qui seules justifient désormais la décision d'adopter telle ou telle forme de langage. La décision d'adopter un langage aux caractéristiques «empiristes » ne reflète-t-il pas simplement, alors, un pur préjugé antimétaphysique ? Quant à la question des mathématiques et de ses fondements, Carnap nous donne-t-il vraiment des raisons convaincantes, fussent-elles "pragmatiques », d'abandonner l'idée que cette science a un domaine d'objets propres, d'opter pour le pluralisme logique, et, enfin, de penser que les mathématiques sont analytiques?

En réalité, contrairement à ce qu'ont pu affirmer quelques commentateurs, on trouve ces justifications «pragmatiques » dans les textes de Carnap. Par exemple, au sujet de la distinction entre vérités logiques et vérités factuelles, lorsqu'il explique que, selon lui, une explication de cette distinction est indispensable pour l'analyse logique de la science ${ }^{41}$, ce qui justifie, à ses yeux, la recherche opiniâtre d'une forme de langage dans laquelle cette distinction puisse être rendue parfaitement claire. Ce qui est remarquable, toutefois, n'est pas que Carnap ne prendrait pas la peine, comme on a pu le lui reprocher, de donner des raisons "pragmatiques » convaincantes (fécondité, simplicité, commodité, etc.) - elles sont exposées dans ses écrits - mais plutôt qu'il choisisse de ne pas en faire une question centrale dans son travail philosophique, comme si les décisions pratiques échappaient à toute tentative de théorisation et de connaissance, et relevaient d'un domaine qui ne serait pas susceptible d'une investigation scientifique : celui des valeurs et de la vie; comme si la distinction principielle entre décision pratique et connaissance théorique était corrélative d'une séparation radicale entre vie et connaissance, la première dépassant les limites de ce que la seconde est capable d'embrasser. Dans un passage de la préface de $L a$ Construction logique du monde que nous avons déjà eu l'occasion de citer, Carnap notait la communauté d'esprit entre certains mouvements artistiques de l'époque et sa propre entreprise philosophique, soulignant que « cet esprit introduit la clarté dans tous les domaines, tout en reconnaissant la complexité de la vie qui n'est jamais totalement pénétrable ${ }^{42} »$. Certains commentateurs ont souligné, en s'appuyant sur des déclarations de ce genre,

41. R. Carnap, Introduction to Semantics, Cambridge, Mass., Harvard University Press, 1942, p. xi.

42. Italiques ajoutées. 
l'évidente influence de Nietzsche (qui est cité à plusieurs reprises dans $L a$ Construction logique du monde, ainsi que dans d'autres textes) sur la pensée de Carnap, ce qui pourrait être interprété comme une indication que son projet philosophique ne puisse pas être compris comme s'inscrivant uniquement et simplement dans la tradition des Lumières ${ }^{43}$.

On sait que Carnap a défendu, pendant la plus grande partie de sa vie, une thèse dite «non-cognitiviste » dans le domaine des valeurs, ce qui signifie qu'il n'existe pas, pour lui, de science des valeurs. Cela n'implique évidemment nullement qu'aucune discussion rationnelle ne soit ni possible ni éclairante sur les questions touchant aux valeurs, à la politique, à la morale ou, plus généralement, aux questions qui appellent non une connaissance théorique mais une décision pratique ${ }^{44}-$ y compris celle d'adopter tel ou tel cadre logique de référence, ou celle d'adopter le pluralisme logique et le principe de tolérance. On notera cependant qu'une telle discussion ne semble pouvoir être utile et féconde qu'à la condition que les interlocuteurs s'accordent au préalable sur le langage dans lequel leur examen rationnel pourra se tenir, et qu'ils partagent, en conséquence, une logique commune. Il y a là, à l'évidence, une difficulté véritable.

Carnap, pour sa part, a consacré plus de temps et de textes à chercher à clarifier ou résoudre des problèmes comme ceux de la confirmation, de l'analyticité, de la signification, de la démarcation, de l'unité de la science, de la signification des probabilités, de la logique modale, ainsi que de nombreux autres, qu'à expliquer pourquoi il a jugé plus fécond, utile, important ou opportun de se consacrer précisément à ces problèmes plutôt qu'à d'autres. Contrairement à beaucoup des philosophes contemporains qui discutent du pluralisme logique, il n'a consacré que peu de textes à la question de savoir s'il fallait ou non adopter cette position, et encore moins à la question de savoir si elle est correcte ou incorrecte, vraie ou fausse, précisément parce qu'il ne s'agissait pas pour lui d'une thèse, mais d'une méthode, voire d'une attitude - la tolérance ${ }^{45}-$, dont la justification peut

43. Cf. par exemple Th. Mormann, «Carnap's Boundless Ocean of Unlimited Possibilities : Between Enlightenment and Romanticism », in P. Wagner (éd.), Carnap's Ideal of Explication and Naturalism, Basingstoke, Palgrave Macmillan, à paraître. Nous ne pouvons entrer ici dans cette question controversée. Sur la place de Carnap entre romantisme et philosophie des Lumières, cf. également l'ouvrage d'André Carus déjà cité.

44. «Durant toute ma vie, depuis mon enfance jusqu'à aujourd'hui, j'ai toujours eu un intense intérêt pour les problèmes de morale, à la fois ceux qui concernent la vie des individus et, depuis la Première Guerre mondiale, ceux de la politique. [...] Nous tous, dans le Cercle de Vienne, avions un fort intérêt pour les événements politiques dans notre pays, en Europe et dans le monde. Ces problèmes étaient discutés en privé, non dans le Cercle, qui était consacré aux questions théoriques. », R. Carnap, «Intellectual Autobiography», in Schilpp (éd.), op. cit., p. 82-83.

45. «Soyons circonspects quand il s'agit de faire des assertions et critiques quand il s'agit de les examiner, mais tolérants quand il s'agit d'autoriser des formes linguistiques ». R. Carnap, «Empirisme, sémantique et ontologie » (1950 ; seconde édition, avec corrections, 1956), trad. F. Rivenc et Ph. de Rouilhan, in R. Carnap, Signification et nécessité, Paris, Gallimard, 1997, p. 335 . 
certes être éclairée - comme toute décision - par des connaissances théoriques, mais non fondée sur elles. Les raisons de ses choix sont montrées, plutôt que démontrées, et son travail est en grande partie exploratoire. Aussi serait-il vain de chercher à comprendre le pluralisme logique et le principe de tolérance, tels que Carnap les entend, en les ramenant à des thèses qui relèveraient purement et simplement de la philosophie de la logique. Pluralisme logique et tolérance ne sauraient être séparés du projet empiriste général qui a orienté l'ensemble de l'œuvre philosophique de Carnap. 